\section{The biodiversity value}

Keywords: Biodiversity, forestry, agriculture, ecosystem, species diversity

\section{Introduction}

In one square kilometer of forest or ocean of an ecosystem, the biotic component includes a wide diversity of animal and plant species, with some dominant species in an ecosystem. A few species are associated with a large number of rare species. As example, a forest of noble wood can contain 50 species of trees, of which half a dozen or even less could add $90 \%$ of the wood. Equally, if a vegetation sample is taken from a meadow, it can contain 30 species of grass, while it would not be surprising if one of the species added $25 \%$ of the total biomass, one third of the species would total more than $80 \%$ and the another two thirds will add only $20 \%$. The distribution can be even more unequal when treating samples of animal life, especially of small organisms. Nonetheless, such distribution pattern does not mean that the less common species are less or not important for the determination and maintenance of the characteristics of an ecosystem. Another characteristic of biodiversity is that diversity is relating to the size of the organism. In small organisms, the diversity is greater than in large ones. For example, in one forest, there is a greater variety of mites comparing with mammals. Besides, the degree of the diversity also depends on the environmental conditions where the living organism lives. For example, there are less diversity in the places, where the living conditions are severe, or they are geographically isolated, such as an island or the Arctic.

The human activities reduce and exert a selective pressure on biodiversity. The economic activities of the primary sector, such as agriculture, forestry, fishing, etc., try to modify the ecosystem. Economic purposes include the transformation of a crop species into the dominant species in an agricultural field. However even in similar situations the diversity tends to persist. When the control measures through the herbicide or weed are not applied, even a grain field demonstrates that a variety of cereal is possibly associated with 10 other species of herbaceous plants with a share of about $7 \%$ of the existing joint biomass. According to the natural diversification trends, it is not rare that where control over the growth of other unwanted species is used, big amount of energy is required in the form of subsidies to prevent such natural growth. So, given this interdependence, the elimination of certain species could alter in this way the conditions necessary to the existence even for the protected species (such as in the case of agricultural crops or floriculture and others), so that the latter, to survive and grow, may needs an energy subsidy directly or indirectly from the outside of the ecosystem, as a high need for energy in the form of mechanical or chemical work in agricultural field.

The impacts of human being on the biodiversity nowadays is a major aspect of the conflict between the economic activities and the ecosystem. The economic system has given a rise to new limiting factors such as pollution and inorganic and non-biodegradable waste flowing into the ecosystem. Chronic overdoses of insecticides in agriculture or forests or the wastewater and industrial waste discharged into streams affect the species composition of ecosystem. The new limiting factors might destroy the needing conditions for the existence of many species and leading to decrease the biodiversity
Volume I Issue 4 - 2017

\author{
Jalil Barkhas \\ University of Granada, Spain
}

Correspondence: Jalil Barkhas, University of Granada, Granada, Spain, Email jbarkhas@ugr.es

Received: November 0I, 2017 | Published: November 28, 2017

accompanied by a demographic boom of the surviving species of the ecosystem. The biodiversity index in fact, is a measure of the degree of the pollution caused by the impact of economic activities on the ecosystem.

Now, for the elaboration of biodiversity index, two aspects need to take in consideration: 1) the component of species diversity and 2) the inequality component or the relative abundance distribution. The component of diversity, defines by the absolute number of species in the field of life forms for which the index defines. From the other side, the inequality component defines by the distributional inequality of importance throughout the different species, given by their respective availability in terms of measurement, such as the amount of existing biomass. The maximum diversity of a domain is established by the combination of the maximum number of variety of species with the perfectly balanced distribution of the weighting coefficient of the importance given by, say, biomass. The two biodiversity indices which follow are alternatively used to measure the degree of diversity by including the two aspects of diversity.

If $\mathrm{n}$ is the number of species and pi the part of existing species in the total biomass, as in:

$$
R_{i}=\left(D_{i}+U_{i}\right) \frac{\Delta P_{i}}{C_{i}}, \text { for all of } n
$$

$\mathrm{p}_{\mathrm{i}}$ will tend to zero as $\mathrm{n}$ increases and $\mathrm{p}_{\mathrm{i}}$ will be approximately equal in value for different $i$. The measure of $\sum_{i=1}^{n} P_{i}^{2}$, called the Simpson index, would give us a measure of biodiversity. On the other side, $\log p_{i}$ grows negatively as the fraction $p_{i}$ decrease. Therefore, the negative of the mean of $\log p_{i}$, that is,

$$
H=-\sum_{i} P_{i} \quad \log _{e} p_{i}
$$

Called the Shannon index, would provide an alternative measure of biodiversity. To get a normalized value of diversity with maximums and minimums of 1 and 0 , respectively, we end with the choice of the following measure $\bar{D}=1-D: \bar{H} \frac{H}{\log _{e} n}$; the minimum will correspond to a situation where $\mathrm{n}=1, \mathrm{p}_{\mathrm{i}}=1$, while the maximum will 
correspond to a situation where $n$ tends to infinity and the values of $p_{i}$ are approximately equal, tending to zero. ${ }^{1}$

Nonetheless, the short and medium term wills are those that have induced the people to maximize the yield of the desired species using their knowledge base and providing energy subsidies. This optimization has often led to monoculture and the elimination of many other species from the surrounding area. Biodiversity should be evaluated as a resource with a great option value. Some of the biotic species without use value today can be considered of immense value for industrial, medicinal or other objectives in the future thanking to the development of the knowledge base. In the 21 st century, with the huge potential contribution of biotechnology to the process of sustainable development and the emergence of innovative products of great importance for well-being, biodiversity has a high value of preservation.

The basic dilemma in the analysis of the preservation of biodiversity comes from the absence of a feasible framework of cost-effectiveness from which, at least in principle, can be raised and discuss basic issues. Most of the current discussions about endangered species completely lack a theoretical framework that can guide policy. The first underlying issue would be the definition of the essential priorities to maintain or increase diversity and prioritize the options of the project for the preservation of biodiversity. The approach of Metrick \& Weitzman ${ }^{2}$ is an interesting example to deal with this problema. ${ }^{2}$ If the unit of analysis is at the species level, the overall value of a species would be the sum of the two components that contribute to the objectives or the rational preservation of: 1) the direct utility of the species and 2) the diversity added by the genes of the related species. The latter is the source for option values, while the former provides use value and contributes to survival. A project to preserve the biodiversity would improve the survival of the species. The latter would be measured through the difference in the probability of survival between presence and absence of measures. The expected gain of undertaking preservation measures would be the sum of the direct utility $\left(\mathrm{U}_{\mathrm{i}}\right)$ and the diversity value in terms of distinction $\left(\mathrm{D}_{\mathrm{i}}\right)$ multiplied by the improvement of the possibility of survival $\left(\Delta \mathrm{p}_{\mathrm{i}}\right)$. This gain is measured against the cost of preservation of $i^{\text {th }}$ species $\left(\mathrm{C}_{\mathrm{i}}\right)$, which is measured in terms of opportunity cost of the increase in protection of $\mathrm{i}^{\text {th }}$ species. Hence

$$
R_{i}=\left(D_{i}+U_{i}\right) \frac{\Delta P_{i}}{C_{i}}
$$

would provide the cost-benefit ratio that helps to order the alternative biodiversity projects.

The resolution of the problem in quantifying factors-the usefulness of a species, the distinctiveness of a species, the change in the survival of a species and the cost of increasing survival-is possible through some powers that represent these factors; For instance, a taxonomic classification of species in addition to the record of the size of a typical member can serve to determine the associated direct utility; if the length and popularity of the fauna or of the animal species -mega-charismatic fauna- are the determining factors of direct utility. The conservation of the biodiversity can be analyzed by relating powers with dependent variables as priority or magnitude of effort of preservation with the various imitations or powers that condense the essence of the four determining factors after prioritization. More research needs on the actions for the preservation of biodiversity, since the laws of endangered species are approved and applied in various countries for a better quantification of the fundamental factors after the elections of preservation of biodiversity.

For an ecosystem the biodiversity is an important issue of economic choice due to its docility vis-a-vis the cost-benefit analysis. The matrix of species assigned by the variety and the weighting coefficient of importance is adapted to the strength and variety of the available contribution of energy and other materials in given climatic and geographical circumstances. Nature maximizes biodiversity, given the constraints of resources, climate and geography, but not at the expense of reducing the energy efficiency of the ecosystem. In fact, the biodiversity relates with the resilience and stability of an ecosystem, which have a positive relationship with the well-being of the structure of existing species, including the human being.

Differently from the way in which physicists define equilibrium after any disturbance, for stability, ecologists want to refer to a reversal of the structure and function of nature to the original system after an exogenous shock experience. In an ecosystem with low biodiversity and concentrated specialized structure, there is a more efficient exploitation of energy and relatively abundant nutrients than in a situation of greater biodiversity and dispersed structure. However, a low-diversity, high-energy ecosystem will tend to boom and burst if the ecosystem is not replenished with the nutrients quickly depleted by the boom, as seen in the growth and decline of algae blooms in a lake, or in the rise and fall of the yield of an agricultural field that produces a single crop. In a strained situation of energy and resources with high biodiversity, on the other hand, most of the energy and nutrient resources are stored in the biomass of the different species. As a result, the rate of withdrawal of said energy or said ecosystem resources would be relatively moderate, allowing the necessary balance between regeneration and withdrawal of resources, resulting in a more stable system. In an unstable situation an ecosystem can develop permanently in a new ecological regime, causing problems of uncertainty related to the future state of primary productivity and the possible survival of certain species. Since the sustainability of economic development would require a stable behavior of the ecosystem, biodiversity becomes a very important condition of sustainable development, apart from being a valuable resource with great option value. The important and positive contribution of biodiversity to ecosystem resilience is an indirect utility or a use value, which should not be ignored.

\section{Acknowledgements}

None.

\section{Conflict of interest}

Author declares there is no conflicto of interest in publishing the article.

\section{References}

1. Odum EP. Ecology: the link between the natural and social sciences. Oxford \& IBM Publishing, New Delhi, India; 1975.

2. Metrick A, Weitzman ML. Conflict and choices in biodiversity preservation. Journal of Economic Perspectives. 1998;12(3):21-34. 